\title{
Applications pratiques de la base de données CANDHIS de mesures d'états de mer in-situ
}

\author{
Luc Hamm $^{\mathrm{a}}$, Gérard Goasguen ${ }^{\mathrm{b}}$ \\ ${ }^{a}$ Directeur Technique, SOGREAH Maritime, 6 rue de Lorraine, 38130 Echirolles. Tél : 07633 4188, \\ mèl:luc.hamm@sogreah.fr \\ ${ }^{b}$ Ingénieur des T.P.E., CETMEF, Technopole Brest-Iroise, BP 5 - 29280 Plouzané. Tél : 029805 6765, \\ mèl :gerard.goasguen@cetmef.equipement.gouv.fr
}

\section{Résumé}

Ce papier présente un certain nombre de traitements de mesures issues de la base de données de houle CANDHIS permettant de déboucher sur des informations pratiques concernant le climat d'agitation d'un site, la documentation des tempêtes et la calibration de modélisations numériques. Il est illustré par l'exploitation de mesures effectuées au large du port du Havre en baie de Seine.

\begin{abstract}
$\underline{\text { Abstract }}$
This article describes analysis of field data, collected in the National Collecting Center of In-Situ Sea State Data (CANDHIS) database, for practical purposes including: the meteorological regime of a site, storm documentation and calibration of hindcast modelling. These analysis are illustrated with measurements performed in the Channel offshore to the port of Le Havre.
\end{abstract}

\section{Présentation de la base de données}

\subsection{Qu'est-ce que CANDHIS ?}

Gestionnaire du principal réseau de mesures in-situ de houle en France, le CETMEF archive dans sa base de données CANDHIS (Centre d'Archivage National de Données de Houle In-Situ) les paramètres d'états de mer issus des mesures réalisées par différents organismes à l'aide de bouées directionnelles ou omnidirectionnelles le long des côtes de France métropolitaine et à l'outremer.

Les informations spécifiques à chaque site instrumenté sont disponibles (site Internet: http://www.cetmef.equipement.gouv.fr/donnees/candhis/home.php) par simple interrogation de la base de données soit en temps réel pour connaître l'état de la mer au jour le jour soit en différé pour accéder à l'ensemble des données disponibles.

Ce service opérationnel depuis maintenant le milieu des années 90 rassemble sur certains sites un nombre appréciable d'années de mesures utilisables dans les applications pratiques.

Nous proposons donc de présenter dans cette communication, les possibilités d'utilisation de cette base de données à travers l'exemple principal des mesures collectées sur la bouée LHA placée à l'entrée du chenal d'accès au port du Havre. Auparavant un bref résumé du contenu de la base est fourni.

\subsection{L'acquisition des mesures}

Cette acquisition varie en fonction du type d'instrument ainsi que des capacités de stockage des données. C'est ainsi que la fréquence d'acquisition varie entre $4 \mathrm{~Hz}$ (Triaxys) et $1,28 \mathrm{~Hz}$ (Datawell directionnelle). La période d'acquisition s'effectue sur des périodes de 20 à 
30 minutes avec une répétition initialement choisie (années 1990) à trois heures et ayant évolué vers une heure actuellement voire une demi-heure sur les matériels les plus récents.

\subsection{Contenu de la base de données}

Une liste très exhaustive de paramètres associant les besoins de l'ingénierie et ceux de la recherche sont stockés dans la base. Cela inclus 35 paramètres pour les mesures omnidirectionnelles auxquels s'ajoutent 4 paramètres directionnels. La définition de ces paramètres est conforme aux recommandations AIRH/AIPCN (1986). Les paramètres omnidirectionnels rendent compte des diverses hauteurs de vagues représentatives dans le domaine temporel et le domaine spectral avec les périodes associées mais aussi des paramètres liés à la largeur du spectre, aux groupements de vagues ainsi qu'aux nonlinéarités. D'autre part, les spectres en fréquence sont également stockés sur une gamme de 64 fréquences comprises entre 0 et $1 \mathrm{~Hz}$ avec un pas variable.

\subsection{Mesures à la bouée LHA}

La bouée-phare LHA marque l'entrée du chenal d'accès au port du Havre (coordonnées $49^{\circ} 31^{\prime} 22 \mathrm{~N}, 0^{\circ} 09^{\prime} 54 \mathrm{~W}$ ) à $17 \mathrm{~km}$ au large du cap de la Hève par fonds de $-17 \mathrm{~m}$ environ. Cette bouée est équipée d'un anémomètre et d'un capteur omnidirectionnel de houle depuis l'automne 1996. Elle est soumise à une agitation complexe incluant des houles résiduelles de l'Atlantique, des mers de vent levées en Manche Ouest ou en Manche Est ainsi que des mers de vent locales générées en baie de Seine.

Des contrôle de cohérence entre les paramètres temporels et spectraux ont été effectués et ont permis de montrer une très bonne corrélation entre $\mathrm{Hmo}$ et $\mathrm{H} 1 / 3$ (fig.1) avec $\mathrm{Hmo}=1,04$ H1/3 ainsi qu'entre T02 et Tav (égalité des deux paramètres). Par contre la corrélation entre $\mathrm{Tp}$ et TH1/3 est beaucoup moins bonne (fig. 2) à cause des états de mer complexes (superposition de mer de vent et de houle)

Rolatlon HmolH1/3 - boudo LHA polit 07603

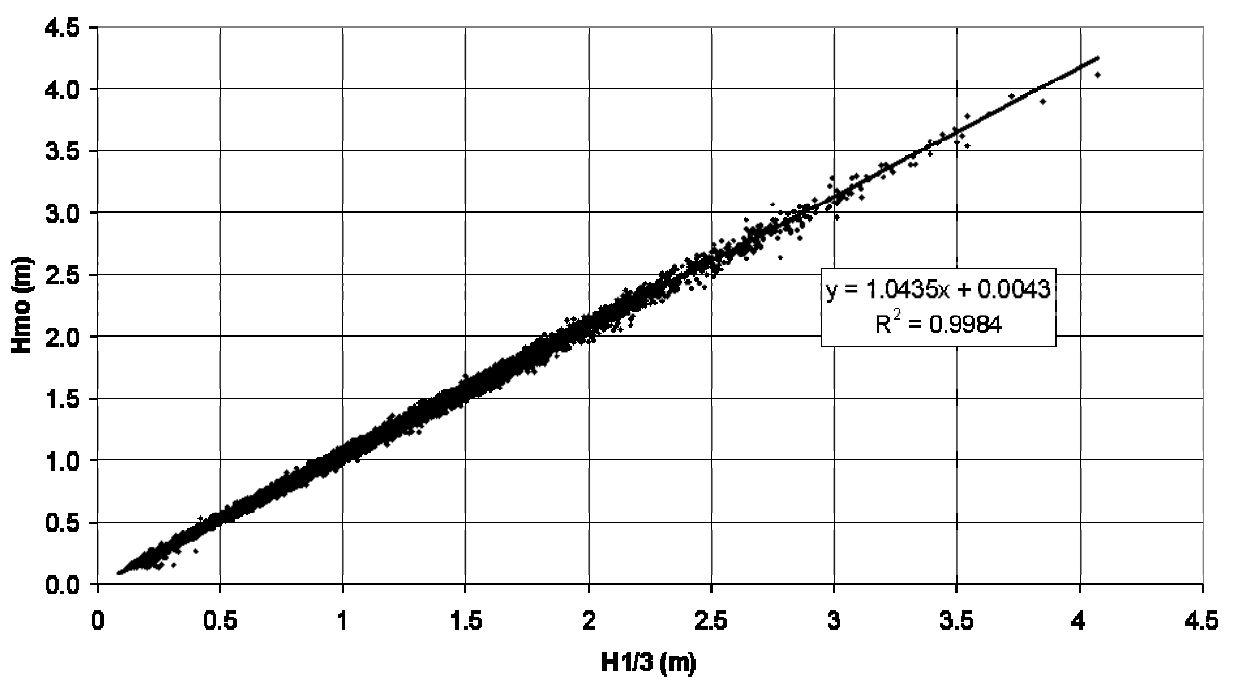

Figure 1: Corrélation entre Hmo et Hl/3 


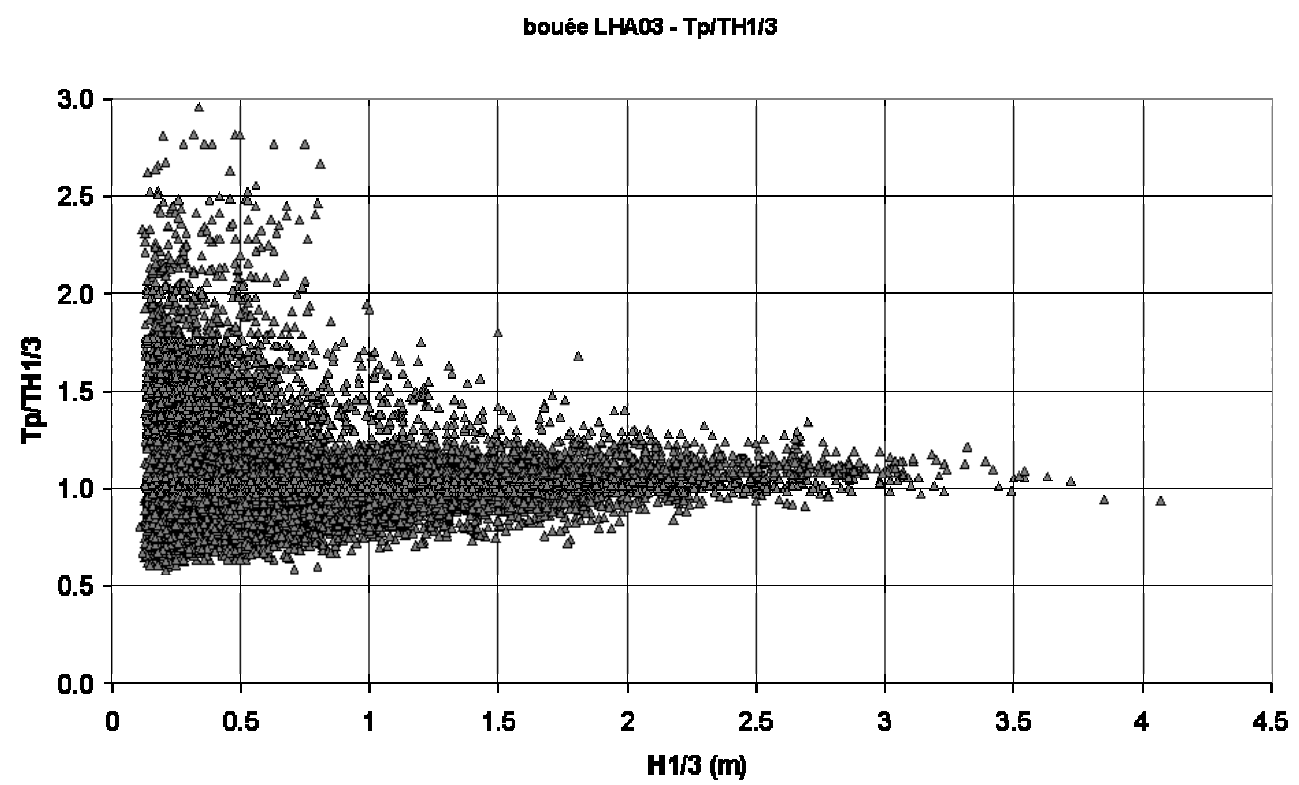

Figure 2: Corrélation entre (Tp/TH1/3) et H1/3

\section{Climat d'agitation}

L'établissement du climat d'agitation sur un site à partir d'une série temporelle couvrant plusieurs années de mesures permet d'étudier les temps d'indisponibilité lors des études de conception portuaire mais aussi d'estimer les périodes d'intempéries et de calme pour les entreprises effectuant des travaux à la mer en détaillant le résultat par saisons. Ainsi pour les travaux de construction de Port2000 au Havre, l'analyse de la bouée LHA entre 1998 et 2001 a permis d'estimer que la tempête annuelle estivale (mois de juin, juillet et août) ne dépassait pas $2 \mathrm{~m}$ de hauteur significative alors que la valeur hivernale a été estimée à $3,6 \mathrm{~m}$. Les mesures des années 2002 et 2003 n'ont pas remis en cause cette estimation permettant ainsi la programmation d'opérations délicates pendant l'été.

L'étude du régime sédimentaire d'un littoral peut également utiliser de telles données à la condition expresse de disposer également de la direction moyenne associée à l'état de mer ce qui milite en faveur du développement de la mesure par bouées directionnelles.

D'autre part, il faut signaler la bonne fréquentation de la partie «mesures en temps réel » du site internet, dans laquelle les mesures effectuées sur les dernières heures sont mises à disposition des professionnels de la mer (pêcheurs, entreprises de travaux publics, plaisanciers, ...). Le nombre de visiteurs différents par mois est de l'ordre de 4500.

\section{Documentation des tempêtes}

La documentation des tempêtes constitue un intérêt majeur de la mesure in-situ. C'est pourquoi, une procédure particulière de déclenchement de la mesure au dessus d'un seuil ajustable selon les sites avait été progressivement mise en place permettant de passer d'une mesure tri-horaire à une mesure horaire afin de capter correctement les pics de tempête. Actuellement, une mesure horaire est systématiquement réalisée grâce aux . progrès réalisés dans la capacité des mémoires de masse. 


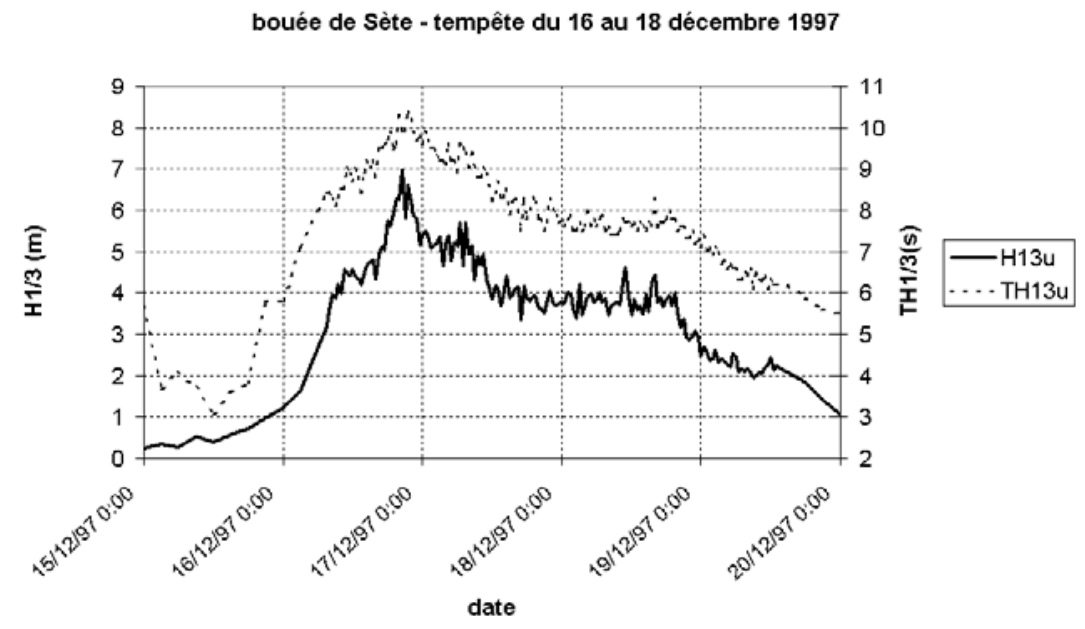

figure 3: Hauteurs significatives mesurées à la bouée de Sète pendant la tempête de 1997

Lorsqu'une collection suffisante de tempêtes peut être rassemblée, la méthode du renouvellement peut alors être utilisée afin d'estimer les temps de retour d' évènements exceptionnels. Ainsi, par exemple, la hauteur significative enregistrée au plus fort de la tempête du 16 décembre 1997 dans le golfe du Lion a atteint la valeur de 6,98 $\mathrm{m}$ à la bouée de Sète (fig. 3). L'extraction et l'analyse de 21152 enregistrements, mesurés entre le 21 octobre 1988 et le 31 décembre 1997 ont pu être utilisés juste après la tempête pour estimer la période de retour d'un tel événement qui s'est avéré être d'une occurrence supérieure à 50 ans.

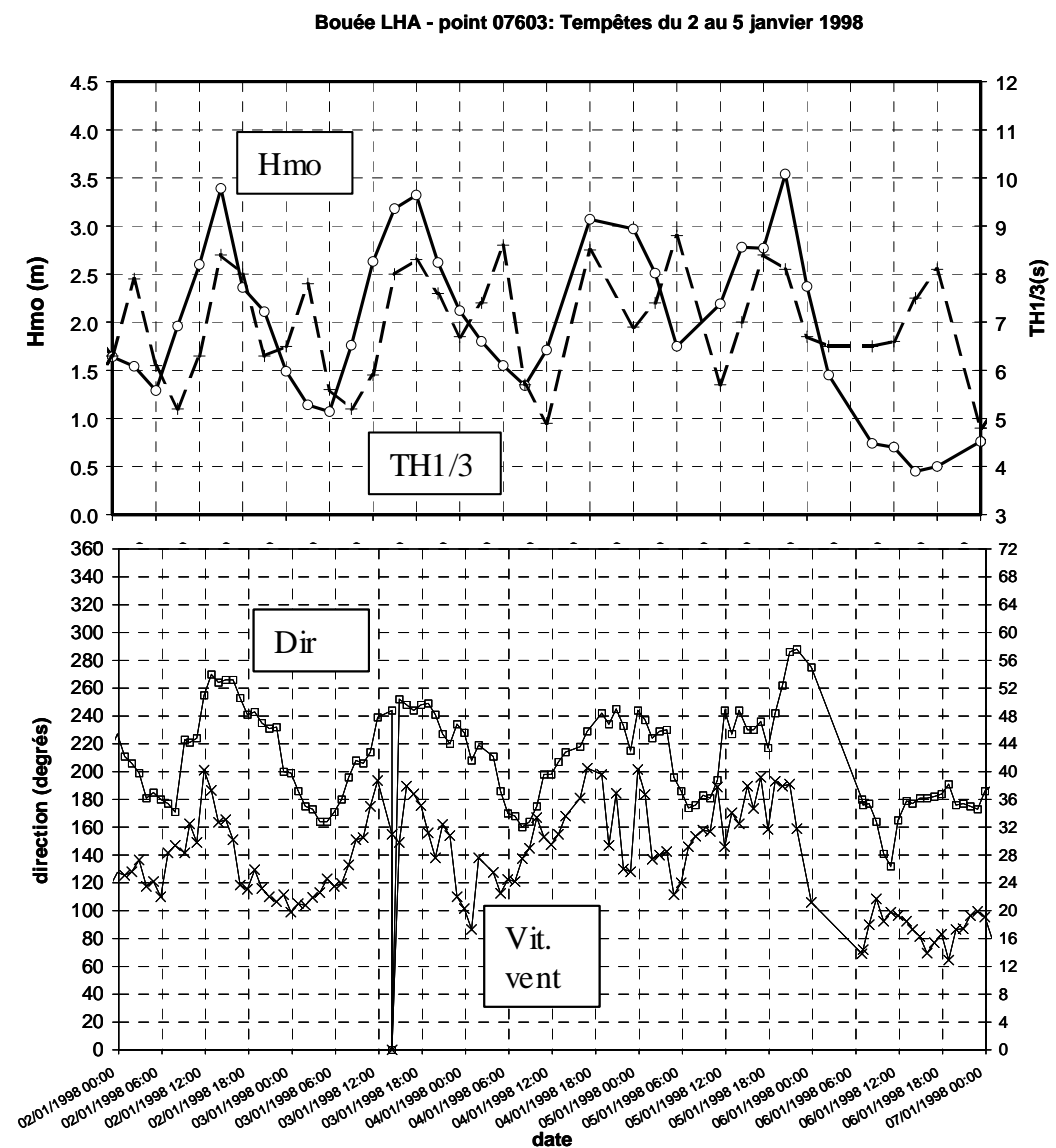

Figure 4. succession de tempêtes en baie de Seine (janvier 1998) 
La figure 4 présente un exemple d'une succession de 4 tempêtes mesurées à la bouée LHA entre le 2 et le 6 janvier 1998. Nous avons associé ici les mesures d'agitation avec celles du vent afin d'avoir une indication sur la provenance de ces états de mer. Une corrélation claire entre le vent et les vagues apparaît sur cette figure avec des pics de tempêtes pour des directions de vent comprises entre N240 et N280.

\section{Validation des modélisations}

\subsection{Le modèle de Rayleigh}

Ces mesures peuvent être également très utiles pour caler un certain nombre de modèles relatifs aux états de mer utilisés usuellement dans l'ingénierie. Un des modèles les plus courants est celui de Rayleigh qui permet de passer d'une simple mesure de Hmo (mesure satellitaire par exemple) à la distribution des vagues de l'état de mer (H1/3, H1/10, H1/100 et Hmax). Ces vérifications faites depuis une vingtaine d'années portent aujourd'hui plus particulièrement sur les vagues anormales lorsque le rapport Hmax/H1/3 dépasse 2.

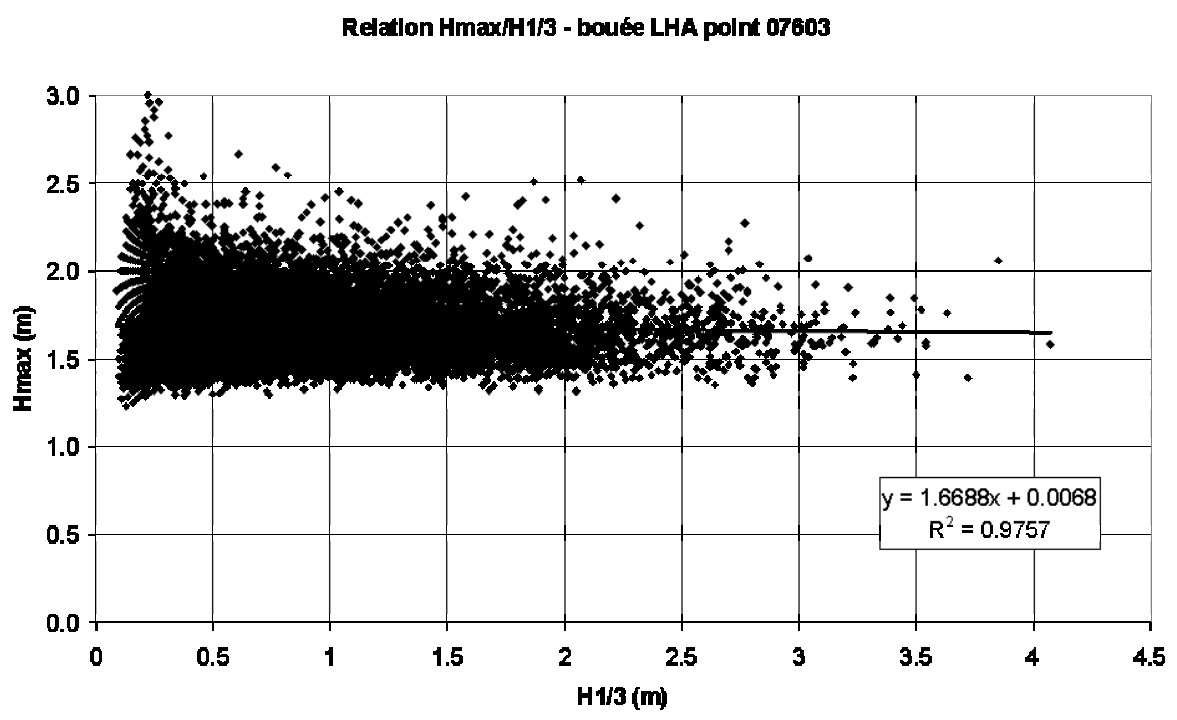

Figure 5. Rapport Hmax/H1/3 à la bouée LHA

L'analyse faite sur ce paramètre à la bouée LHA confirme la valeur moyenne de 1,6 généralement admise pour des enregistrement de 20 minutes. Elle montre cependant de grands écarts avec des valeurs comprises entre 2,0 et 2,5 y compris pendant certaines tempêtes.

\subsection{Les formules simplifiées de génération des mers de vent}

Les formules simplifiées de génération dites formules de fetch sont également très utilisées en ingénierie côtière pour générer de longues séries temporelles d'états de mer sur des sites où le fetch est confiné (baies, estuaires) et lorsqu'il reste inférieure à $100 \mathrm{~km}$. De nombreuses formules ont été publiées depuis 50 ans. Elles ont fait l'objet d'une étude comparative à la bouée LHA en sélectionnant les états de mers associés à des vents de secteur N220 à N270.

L'approche la plus pertinente pour rendre compte des mesures s'est avérée être celle de Kahma et Calkoen(1992) qui introduit la notion de stabilité de l'interface air-mer comme étant un facteur essentiel pour estimer les états de mer générés par le vent. 
Ils proposent ainsi deux formulations différentes : une expression donnant une fourchette basse de la hauteur significative spectrale lorsque l'interface est stable et une expression donnant une estimation haute lorsque l'interface est instable. La figure 6 indique que ces deux formules encadrent bien les mesures effectuées avec notamment une série de tempêtes levées par une interface instable.

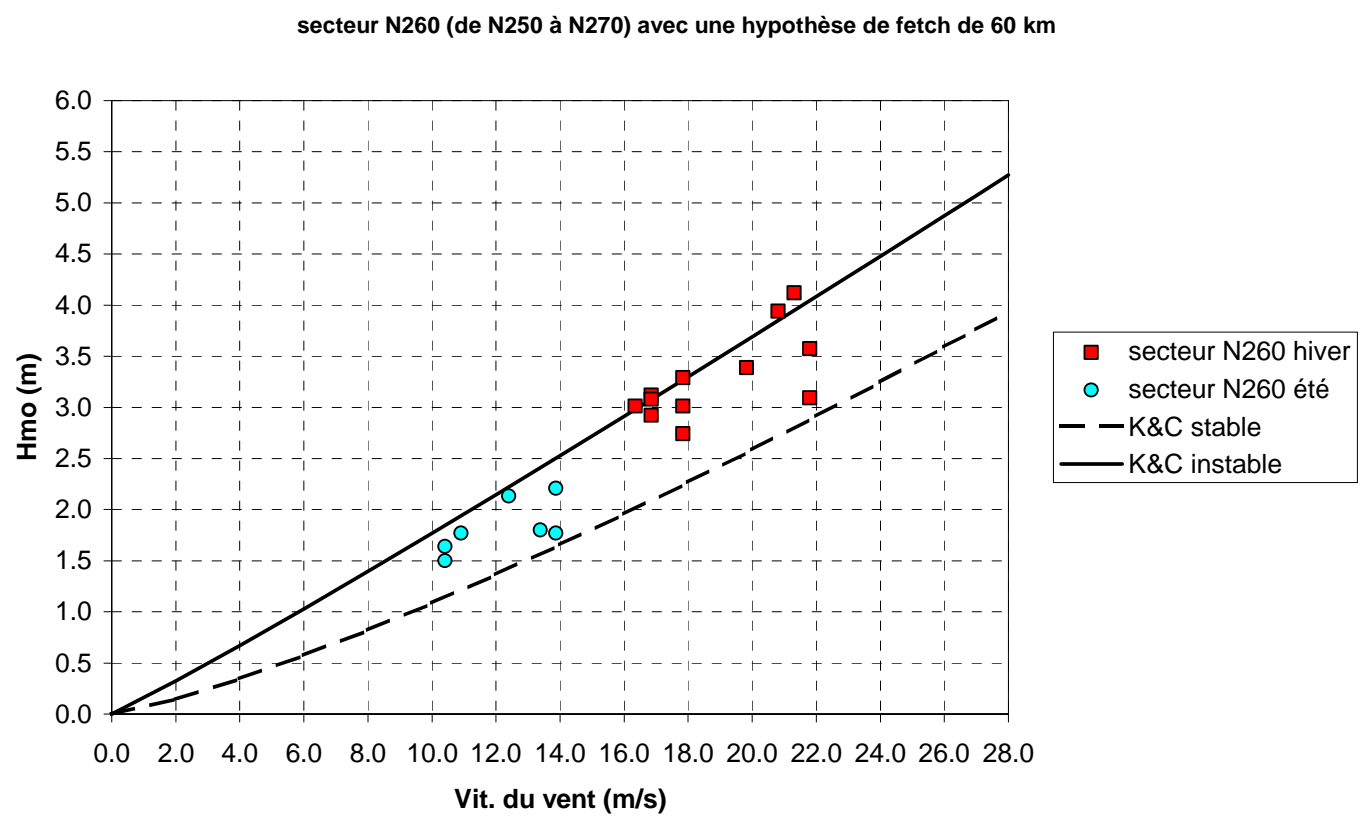

Figure 6. Application des formules de Kahma et Calkoen (1992) aux mesures effectuées à la bouée LHA pour le secteur N250 à N270

\subsection{La reconstitution des états de mer}

Les mesures en mer sont également indispensables pour caler et valider les modèles numériques de reconstitution d'états de mer qui constituent à l'heure actuelle l'outil le plus complet pour établir le climat d'agitation sur un site. De tels modèles peuvent être construits soit à l'occasion d'une étude particulière, soit dans un cadre d'intérêt plus général. Nous citerons en particulier le projet «Atlas numérique de houle » d'intérêt national en cours de réalisation par un partenariat entre le CETMEF, le LNHE (EDF R\&D) et Météo-France (Toulouse) qui vise à fournir une simulation continue sur 20 ans des états de mer le long des côtes de l'Atlantique, la Manche et la Mer du Nord.

\section{Conclusions: Limitations actuelles et perspectives de développement}

Le génie côtier a un besoin constant d'estimation des conditions d'agitation le long du littoral. Ce besoin nécessite à l'amont l'accès à des bases de données complètes et fiables sur une période de temps suffisante allant de 5 ans pour les suivis de travaux à la mer jusqu'à 20 ans (voire 40 ans) pour le dimensionnement d'ouvrages par exemple.

Après avoir utilisé pendant près de 30 ans les observations visuelles de navires sélectionnées ou de sémaphores (1960-1990), les bureaux d'études se sont tournés au milieu des années 90 vers la mesure satellitaire. Celle-ci a cependant montré ses limites au bout de quelques années d'utilisation conduisant à privilégier actuellement la reconstitution d'états de mer par la modélisation numérique. 
A chaque étape, la mesure par bouée in-situ est restée une référence irremplaçable et le juge de paix pour la calibration des instruments de télédétection ou la validation des modélisations numériques. Cela a conduit au développement de réseaux permanents de mesures le long des côtes des principaux pays industrialisés (USA, Japon, Royaume-Uni, Espagne, Italie,...). La mise en place de CANDHIS en France participe à cette dynamique et les quelques applications pratiques présentées ici ont montré le potentiel de ces données aussi bien pour la fourniture de données en temps réel que pour les études historiques.

Le Cetmef envisage de développer ce service selon deux directions : D'une part, il est prévu d'enrichir le site internet par la mise en ligne de nouveaux produits comme les graphes mensuels de variations des hauteurs significatives et l' estimation des houles annuelle et décennale. Pour les capteurs directionnels, il est également prévu de fournir des histogrammes de directions et des corrélogrammes hauteur-direction et direction-période.

D'autre part, il s'agit d'améliorer la description des états de mer complexes par la décomposition en systèmes de vagues homogènes ( mers de vent; houle primaire, voire houle secondaire) comme cela existe actuellement (sous forme approximative) dans les observations visuelles des navires sélectionnés.

Sur ce second point, des développements significatifs sont encore nécessaires. Pour les observations omnidirectionnelles, seul l'examen visuel du spectre en fréquence permet actuellement de conclure sur la présence d'un (voir fig. 7) ou plusieurs systèmes de vagues (voir fig. 8)

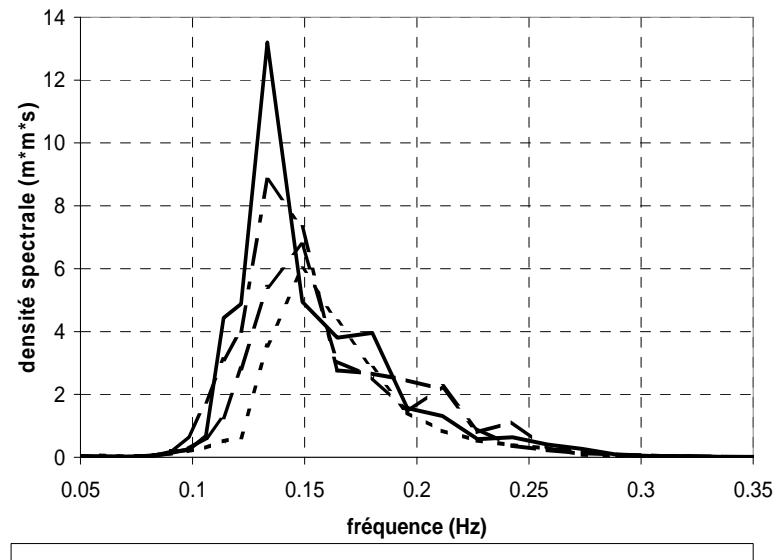

- - 10/10/2000 01:00 - 10/10/2000 02:00 - 10/10/2000 03:00 - 10/10/2000 04:00

Figure 7. Développement simple d'une mer de vent à la bouée LHA(tempête du 10 Octobre 2000)

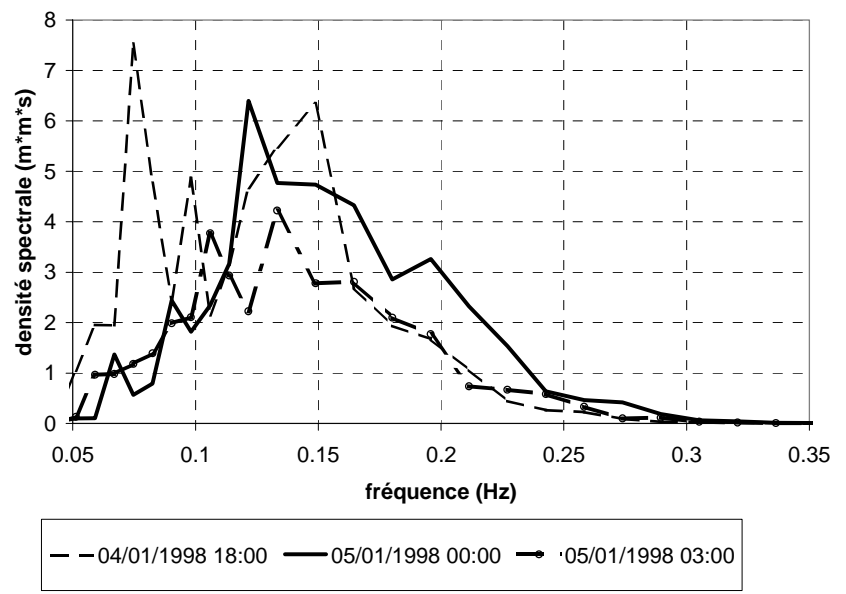

Figure 8. Etat de mer complexe à la bouée LHA (tempête du 4/5 janvier 1998) 
Des critères empiriques reposant sur la largeur du spectre ou la relation entre les différentes périodes pourraient permettre de repérer automatiquement ces états de mer complexes.

Pour les observations directionnelles, la tâche est encore plus ardue. Les travaux de Benoit sur le sujet (voir par exemple Benoit et Goasguen, 1996) devraient permettre d'aboutir à une telle séparation.

Enfin, une exploitation plus approfondie de l'ensemble des données déjà disponibles par des actions de recherche appliquée est également une voie de développement qui reste d'actualité par exemple pour explorer la question de l'impact de l'instabilité de l'interface airmer sur la génération des mers de vent.

\section{Remerciements}

Les auteurs remercient l'équipe du CETMEF à Brest en charge de la gestion de CANDHIS à savoir : messieurs Guy AMIS, Alain LE BERRE et Michel ROGARD

\section{Références}

1.AIRH/AIPCN (1986). Paramètres des états de mer. Supplément au bulletin $n^{\circ} 52$ de l'Association Internationale Permanente des Congrès de Navigation, Bruxelles, 25 pages

2.Benoit, M. et G. Goasguen (1996). Analyse directionnelle des états de mer. Comparaison de différentes méthodes d'analyse appliquées à des capteurs ponctuels. Revue Technique des Phares et Balises, n97, pp 3-27

3.Kahma, K.K. et C.J. Calkoen (1992). Reconciling discrepancies in the observed growth of wind-generated waves. J. of Phys. Oceanography, vol.22, pp 1389-1405 